\title{
Bioabsorbable interference screw versus bioabsorbable cross pins: influence of femoral graft fixation on the clinical outcome after ACL reconstruction
}

\author{
Stephan Frosch • Anne Rittstieg • Peter Balcarek • \\ Tim Alexander Walde · Jan P. Schüttrumpf • Martin M. Wachowski • \\ Klaus M. Stürmer · Karl-Heinz Frosch
}

Received: 12 April 2011 / Accepted: 30 December 2011/Published online: 31 January 2012

(C) The Author(s) 2012. This article is published with open access at Springerlink.com

\begin{abstract}
Purpose The aim of this study was to evaluate the clinical outcome and differences in anterior-posterior laxity of ACL reconstruction using a bioabsorbable interference screw for femoral graft fixation when compared to femoral bioabsorbable cross pin fixation.

Methods Clinical outcome was evaluated among 59 patients 1 year after arthroscopic ACL reconstruction with hamstrings graft in a prospective, non-randomised study. In 31 cases, femoral fixation of the graft was performed using a bioabsorbable interference screw. In 28 cases, two bioabsorbable cross pins were used for femoral fixation. Patients were evaluated using Tegner, Lysholm and Marshall scores, the visual analogue scale for pain and KT-1000 arthrometer measurement.

Results No significant difference $(P \geq 0.05)$ was observed at follow-up for the knee scores. The average Tegner score was 5.83 points $( \pm 2.00)$ for the interference screw fixation and 5.83 points $( \pm 1.24)$ for the cross pin fixation; the average Lysholm score was $93.58( \pm 5.79)$ to $92.72( \pm 6.34)$ points; and the average Marshall score $46.72( \pm 2.4)$ to $47.30( \pm 2.35)$ points. No significant difference was found for the visual analogue scale for pain. KT-1000 arthrometer measurement revealed a significant $(P<0.05)$ difference in the mean sideto-side anterior translation at all applied forces. At $67 \mathrm{~N}$, the
\end{abstract}

S. Frosch $(\varangle) \cdot$ A. Rittstieg · P. Balcarek ·

T. A. Walde - J. P. Schüttrumpf · M. M. Wachowski ·

K. M. Stürmer · K.-H. Frosch

Department of Trauma Surgery, Plastic and Reconstructive

Surgery, Medical University of Göttingen, Göttingen, Germany

e-mail: Stephan.Frosch@med.uni-goettingen.de

\section{K.-H. Frosch}

Department of Trauma and Reconstructive Surgery,

Asklepios Clinic St. Georg, Hamburg, Germany mean difference was $1.53 \mathrm{~mm}( \pm 1.24)$ in the interference screw group and $0.47 \mathrm{~mm}( \pm 1.18)$ in the cross pin group $(P<0.05)$. At $89 \mathrm{~N}$, the mean differences were $1.85 \mathrm{~mm}$ $( \pm 1.29)$ versus $0.59 \mathrm{~mm}( \pm 1.59)$, respectively, $(P<0.05)$, and maximum manual displacements were $2.02 \mathrm{~mm}( \pm 1.26)$ versus $1.22 \mathrm{~mm}(1.18 ; P<0.05)$.

Conclusions In ACL reconstruction with hamstrings graft, similar clinical results are obtained for the use of bioabsorbable cross pins when compared to bioabsorbable interference screws for femoral fixation. Cross pin fixation was superior with regard to the anteroposterior laxity as measured with KT-1000.

Keywords Milagro screw - Interference screw - Cross pins $\cdot$ ACL reconstruction $\cdot$ Postoperative outcome

\section{Introduction}

In hamstring ACL reconstruction, graft fixation is a critical factor for the healing process. Interference screws as well as cross pins are common intraosseous graft fixation techniques. Good clinical results can be achieved with both devices [1].

Bioabsorbable interference screws and metal interference screws are equally successful in graft fixation [5, 7, 20, 23], but bioabsorbable interference screws exhibit advantages due to their biodegradability [6, 8]. Disadvantages of the metal interference screws like graft irritation owing to their sharp edges, problems during potential revision procedures as well as distortion of MRI have led to the preference for bioabsorbable screws in ACL fixation $[9,14,30]$.

Advantages of interference screws are as follows: (1) Reduced early motion of the graft within the tunnel, which is important for stable healing [12]; (2) Less synovial fluid in the bone tunnel, reducing possible negative effects of cytokines 
[13]; and (3) Avoiding the so-called bungee effect due to fixation at a point close to the tunnel entrance [13].

Disadvantages of interference screws are possible graft irritation caused by introducing the screw, a reduced bonetendon interface and a reported slippage of the graft causing clinical failure [10, 22, 24].

A lack of replacement of bioabsorbable screws by bony tissue even after an extended period of time is reported [5, $22,24,27,28]$, as well as inflammation of the synovium elicited by foreign body reactions [2, 25, 27].

A new development is the bioabsorbable Milagro interference screw, made of $30 \% \quad \beta$-TCP (tricalcium phosphate) and 70\% PLGA (polylactic-co-glycolic acid). It was shown in a previous study [8] that due to the Milagro screw's material properties, degradation of the screw is up to $90 \%$ after 12 months and well suited to the healing process of the ACL transplant $[4,8,16]$. In the first three to 6 months, when the graft requires stable fixation, Milagro screws displayed almost no resorption $[8,16]$. Moreover, the osteoconductive properties of the material induce bony replacement of the screw, which could be advantageous in revision surgery [8].

Another common device used for ACL fixation is the biodegradable cross pin (RigidFix, DePuy Mitek, Norderstedt, Germany), which is inserted perpendicular to the tunnel and fixate the graft in the tunnel by lancing it. Several studies have shown comparable clinical results and primary stability after ACL reconstruction using either the interference screws or cross pins for securing the graft $[11,17,29]$.

There is no clinical study comparing Milagro interference screw fixation with cross pin fixation on the clinical outcome and anterior-posterior laxity.

The aim of this study is to evaluate the clinical results and translational stability of ACL reconstruction with hamstring tendons using Milagro screws for femoral fixation of the graft and to compare them with the results for femoral cross pin fixation.

The hypothesis of this study is that the Milagro screw shows superior clinical outcomes as well as superior anterior-posterior laxity when compared to the cross pins in ACL graft fixation.

\section{Materials and methods}

This prospective, non-randomised study involved fifty-nine patients who underwent hamstring ACL reconstruction. Two different femoral graft fixation methods were performed. To compare the clinical outcome of each fixation method, we separated the patients into two groups according to the fixation methods used.

Table 1 shows the demographic baseline profile of both groups.
Table 1 Demographic baseline profile

\begin{tabular}{lll}
\hline Variables & $\begin{array}{l}\text { Cross pin } \\
\text { group } \\
N=28\end{array}$ & $\begin{array}{l}\text { Milagro } \\
\text { group } \\
N=31\end{array}$ \\
\hline Female & 10 & 12 \\
Male & 18 & 19 \\
Mean age (years) & $28.2( \pm 8.0)$ & $24.6( \pm 7.2)$ \\
Body mass index (kg/m $\left.{ }^{2}\right)$ & $24.9( \pm 2.9)$ & $24.6( \pm 3.2)$ \\
Mean interval to surgery (weeks) & $11.09( \pm 4.0)$ & $14.91( \pm 3.4)$ \\
Follow-up examination (month) & $12.40( \pm 0.8)$ & $12.45( \pm 1.1)$ \\
Accompanying injuries & & \\
$\quad$ Lateral meniscus & 8 & 7 \\
$\quad$ Medial meniscus & 4 & 3 \\
$\quad$ Chondral lesion (in numbers & 6 & 7 \\
$\quad$ unless otherwise labelled) & & \\
\hline
\end{tabular}

In a period of 1 year, we initially performed thirty-one ACL reconstructions using Milagro interference screws, followed by twenty-eight ACL reconstructions using the cross pin technique for femoral fixation. Tibial fixation in all patients was performed with Milagro interference screws.

Inclusion criteria are the following: (1) primary ACL rupture with the indication for an ACL reconstruction (instability signs like giving way, positive Lachman and/or pivot shift test); (2) closed epiphyseal plates.

Exclusion criteria are the following: (1) the presence of additional fractures around the knee joint; (2) previous surgery on the affected knee joint; (3) cartilage lesions ICRS grade 2 exceeding $3 \mathrm{~cm}^{2}$, cartilage lesions ICRS grade 3 and 4; (4) additional PCL lesions; (5) autologous chondrocyte transplantation; (6) mosaicplasty with more than one transplanted cylinder (or a cylinder $>1 \mathrm{~cm}$ ); (7) more than $50 \%$ of the medial or lateral meniscus resected; (8) patients with axis deformities or underlying diseases that resulted in physical impairment.

Implants for graft fixation

Milagro $^{\mathrm{TM}}$ interference screws (DePuy Mitek, Norderstedt, Germany) are made of 30\% $\beta$-TCP (TriCalcium phosphate) and $70 \%$ PLGA (polylactic-co-glycolic acid). They are available in diameters of 7-12 mm and lengths of 23,30 or $35 \mathrm{~mm}$.

Two Cross pins (RigidFix, Ethicon, Mitek Division, Norderstedt, Germany) were used in diameter of $3.3 \mathrm{~mm}$ and length of $42 \mathrm{~mm}$. They are made of poly-L-lactic acid (PLLA) and were used only for femoral fixation in this study.

Surgical technique

The ACL reconstruction was performed arthroscopically by two experienced surgeons. In all cases, semitendinosus or semitendinosus combined with gracilis tendons were 
used as grafts, depending on the diameter and length of the tendons. The tendons were either trebled or quadrupled to reach a graft diameter of $7-8.5 \mathrm{~mm}$ and a graft length of $9 \mathrm{~cm}$. After removal, the tendons were sutured in a whipstitch fashion and augmented on the femoral and tibial aspects. The tibial tunnel was prepared using an alignment jig, with the footprint of the ACL as reference (Fig. 1). The cortex was opened at an angle of $55^{\circ}$ to the tibial articular surface directly above the pes anserinus.

The femoral tunnel was prepared and drilled over the anteromedial portal using an alignment jig with a $6 \mathrm{~mm}$ offset, related to the anatomical landmarks of the ACL, slightly more oriented to the anteromedial bundle than to the posterolateral bundle (Fig. 2). The tunnel was drilled at $120^{\circ}$ of knee flexion.

In group one, after placing the graft in the tunnel, the Milagro screws were introduced in the femoral tunnel to fix the graft. The graft was pretensioned by moving the knee joint through its range of motion before a tibial interference screw was placed as close to the joint as possible at a knee flexion of $20^{\circ}$. A $23-\mathrm{mm}$ femoral screw and $30-\mathrm{mm}$ tibial screw were used. The bone tunnel diameter was adapted to $0.5 \mathrm{~mm}$ below graft height. The diameter of the Milagro screws was selected according to the bone tunnel diameter.

In group two, two cross pins were used for femoral fixation. A cross pin guide was placed in the tunnel. Two sleeves were inserted in the interlocking trocars and drilled into the lateral side of the femur towards the tunnel. After removing the guide, while the sleeves were left in place, the graft was placed in the tunnel. Then, guided by the sleeves, the cross pins were driven into the tunnel until the pins had crossed the tunnel and both ends were anchored in the bone. The subsequent steps were the same as in group one. Tibial fixation was performed using a 30-mm Milagro interference screw.

\section{Follow-up treatment}

Care following surgery included 6 weeks of partial weightbearing (10-20 kg) with the surgical leg using crutches.

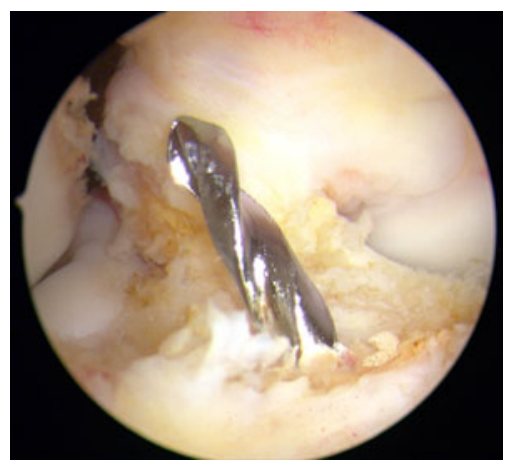

Fig. 1 Arthroscopic picture: The tibial tunnel was placed with the tibial insertion of the ACL as reference (right knee)

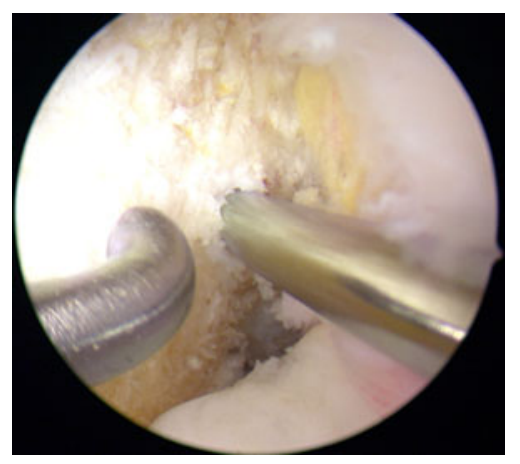

Fig. 2 Arthroscopic picture after ACL reconstruction. The femoral tunnel was placed closer to the origin of the anteromedial bundle than to the posterolateral bundle (left knee)

No brace should be used. The range of motion of the knee should not exceed $0-0-90^{\circ}$ (Ext/Flex) for the first 6 weeks postoperatively.

\section{Clinical evaluation}

The mean follow-up examination of the cross pin group was performed after 12.4 months $( \pm 0.8)$ and for the Milagro group after 12.45 months $( \pm 1.1)$. The clinical evaluation was based on three functional knee scores: the Lysholm score [26], the Tegner score [26] and the Marshall score [18]. The KT-1000 arthrometer measurement was used to evaluate the mean difference in anterior-posterior laxity (compared to the healthy contralateral knee) with a maximum measurement accuracy of $1 \mathrm{~mm}$. The visual analogue scale was used to assess the level of pain during daily living $(0$ points $=$ no pain, 10 points $=$ maximal pain $)$.

Statistical analysis

Functional scores, anterior instability and pain score were compared between the two fixation methods using the nonparametric Mann-Whitney $U$-test. The significance level was set to alpha $=5 \%$ for all test. Analyses were performed using the software Statistica (version 9.1, StatSoft).

\section{Results}

Thirty-nine patients were treated with an ACL reconstruction using cross pins for femoral fixation of the hamstring graft. Eleven Patients were excluded of the study corresponding to the exclusion criteria: two medial meniscus resection $>50 \%$; one lateral meniscus resection $>50 \%$; three cartilage lesions ICRS 3; two additional fractures of the proximal tibia; three previous surgeries on the affected knee joint. Twenty-eight patients were included in the study, and twenty-eight patients participated at the follow-up examination for the cross pin group. 
Thirty-seven patients were treated with an ACL reconstruction using the Milagro screw for fixation of the hamstring graft. Six patients were excluded: two lateral meniscus resection $>50 \%$; three cartilage lesions of ICRS grade 3 and higher; one patient to take part in the study. All of the included thirty-one patients took part at the followup examination.

The follow-up percentage was 100 for the Milagro and cross pin group.

Table 1 shows the demographic baseline profile of both groups.

Functional scores

No significant differences (n.s.) were found between the groups when comparing the femoral fixation methods with regard to the functional scores. The median values of the Lysholm score were 94 (81-100) points in the Milagro group and 94.5 (82-100) points in the cross pin group (Fig. 3). The median values of the Tegner score were 6 (3-9) points versus 6 (4-9) points (Fig. 4). The median values of the Marshall score did not differ significantly between the groups, with 47.5 (43-50) points for group one and 48 (42-50) points for group two (Fig. 5).

\section{KT-1000 arthrometer}

Comparing postoperative stability using the KT-1000 arthrometer revealed a significant difference between the groups $(P<0.05)$. The mean differences in anterior-posterior laxity for the Milagro group versus the cross pin group were the following: for $67 \mathrm{~N} 1.5 \mathrm{~mm}( \pm 1.2)$ versus $0.5 \mathrm{~mm}( \pm 1.2)$; for $89 \mathrm{~N} 1.9 \mathrm{~mm}( \pm 1.3)$ versus $0.6( \pm 1.6)$; and for maximum manual displacement $2.0 \mathrm{~mm}( \pm 1.3)$ versus $1.2 \mathrm{~mm}( \pm 1.2$; Fig. 6$)$.

Visual analogue scale for pain

Patients evaluated their pain during daily living using the visual analogue scale with a mean score of $1.1( \pm 1.5)$ for

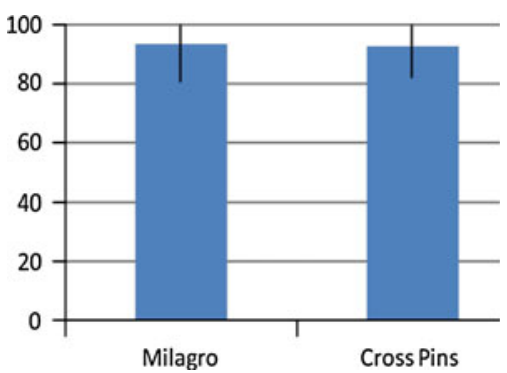

Fig. 3 Lysholm score. No significant difference (n.s.) at the followup between the two groups (median values)

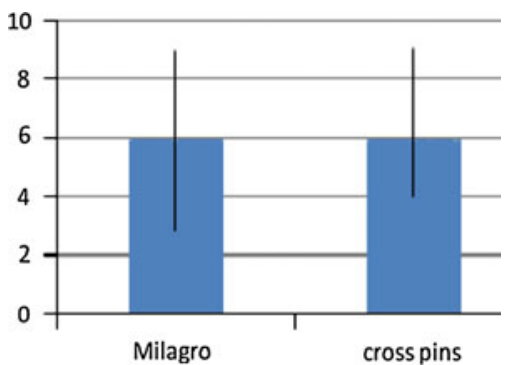

Fig. 4 Tegner score. No significant difference (n.s.) at the follow-up between the two groups (median values)

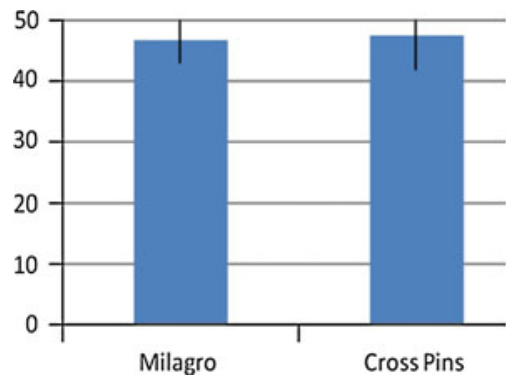

Fig. 5 Marshall score. No significant difference (n.s.) at the followup between the two groups (median values)

the Milagro screw group and $1.4( \pm 1.4)$ for the cross pin group. These results were not significantly different (n.s.).

\section{Complications}

Two patients in the Milagro ${ }^{\mathrm{TM}}$ group required revision surgery due to a cyclops syndrome. No complications were found in the cross pin group.

\section{Discussion}

The most important finding of the present study was that cross pin fixation is superior with regard to the anteroposterior laxity when compared to Milagro screw fixation as measured with KT-1000. Therefore, the hypothesis of this study that the Milagro screw shows superior clinical outcomes as well as superior anterior-posterior laxity when compared to the cross pins in ACL graft fixation must be discarded.

After ACL reconstruction, progressive rehabilitation programmes and demands of patients to participate in sports activities as early as possible require a secure and reliable fixation of the graft. Interference screws as well as cross pins show good clinical results and primary stability after ACL reconstruction [1]. 


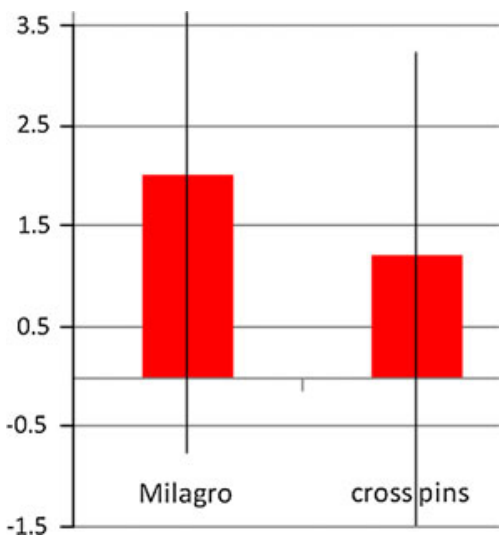

Fig. 6 KT-1000 at max. displacement. Significant difference $(P<0.05)$ at the follow-up between the two groups

During activities of daily living, forces up to $450 \mathrm{~N}$ stress the graft [3, 21]. Zantop et al. [29] examined the initial fixation strength of two 3.3-mm bioabsorbable pins compared to interference screws for hamstring grafts in bovine knees. In the cycle loading test, they found no significant difference under 1,000 cycles to $250 \mathrm{~N}$. Remarkable finding of the study is that only grafts fixed with cross pins survived 1,000 cycles to $450 \mathrm{~N}$.

Harilainen et al. [11] presented a 2-year follow-up randomised trial including 120 patients, comparing cross pin fixation and BioScrew fixation after ACL reconstruction with hamstring tendons. They found no significant difference at the 1- and 2-year follow-up evaluation at the clinical examination, knee scores (Tegner Activity Level, Lysholm, IKDC, and Patellofemoral Scores) and laxity measurements (Lachman, Pivot-shift).

According to these observations, the present study does not find any significant differences (n.s.) at the clinical outcome when comparing femoral cross pin fixation with Milagro screw fixation after ACL reconstruction. The median values of the Tegner score were 6 points (3-9) for the Milagro fixation and 6 points (4-9) for the cross pin fixation; the median values of the Lysholm score were 94 (81-100) versus 94.5 (82-100) points; and the median values of the Marshall score were 47.5 (43-50) versus 48 (42-50) points. The median values of the visual analogue scale for pain during daily living also showed no significant difference (n.s.): $1(0-4)$ points versus $1.5(0-5)$ points.

The KT-1000 arthrometer measurement revealed significant differences $(P<0.05)$ in the mean side-to-side anterior translation at all applied forces. At $67 \mathrm{~N}$, the mean difference was $1.5 \mathrm{~mm}( \pm 1.2)$ in the Milagro group $0.5 \mathrm{~mm}( \pm 1.2)$ in the cross pin group. At $89 \mathrm{~N}$, the mean differences were $1.9 \mathrm{~mm}( \pm 1.3)$ versus $0.6 \mathrm{~mm}( \pm 1.6)$, respectively, and maximum manual displacements were $2.0 \mathrm{~mm}( \pm 1.3)$ versus $1.2 \mathrm{~mm}$ (1.2). These results support the statement of Zantop et al. [31] that interference screws provide a significant inferior biomechanical stability than cross pins do.

Although the KT-1000 arthrometer measurement revealed significant differences $(P<0.05)$ in the mean side-to-side anterior translation, there is no effect on the clinical outcome.

There are limitations of the study. First, some authors state that 1-year follow-up examination after ACL reconstruction might be too short to evaluate the postoperative outcome. In response to that, primary graft healing is completed after 12 months [8]. Direct contact is established between tendon and bone tunnel wall within 12 weeks, and bone-tendon junction takes up to 24 weeks [13]. Patients after ACL reconstruction participate in full contact sports after 7-9 months. Therefore, differences at the clinical outcome, especially differences at the anteroposterior laxity, should be revealed after 12 months. A subsequent study with a 5-year follow-up should be aimed to examine the clinical outcome.

Secondly, rotatory laxity has not been assessed. There are reports that the pivot-shift examination has significant associations with subjective symptoms and function after ACL reconstruction [15]. We did not assess the pivot-shift test, because of a limited comparability due to different muscular tension of the patients during the test and because of a high inter-observer variation [19].

Finally, we did not investigate radiographic outcomes, such as tunnel widening. There are already several studies focusing on tunnel widening after ACL reconstruction. A clinical relevance of tunnel widening could not been shown $[1,8]$.

The findings of this study imply an advantage of the cross pins over the interference screw in ACL fixation, and therefore we use femoral cross pins fixation as a standard procedure in ACL reconstruction.

\section{Conclusion}

In ACL reconstruction with hamstrings graft, similar clinical results are obtained for the use of cross pins when compared to Milagro interference screws for femoral fixation. Cross pin fixation was superior with regard to the anteroposterior laxity as measured with KT-1000.

Acknowledgments The authors declare that they have no conflict of interest. All patients gave their informed consent prior to their inclusion in the study. Special thanks to Dr. Klaus Jung, Department of Medical Statistics-Medical University of Göttingen, for his generous support.

Open Access This article is distributed under the terms of the Creative Commons Attribution License which permits any use, distribution, and reproduction in any medium, provided the original author(s) and the source are credited. 


\section{References}

1. Andersson D, Samuelsson K, Karlsson J (2009) Treatment of anterior cruciate ligament injuries with special reference to surgical technique and rehabilitation: an assessment of randomized controlled trials. Arthroscopy 25(6):653-685

2. Bostman O, Hirvensalo E, Makinen J, Rokkanen P (1990) Foreign body reactions to fracture fixation implants of biodegradable synthetic polymers. J Bone Joint Surg Br 72(4):592-596

3. Brand J, Weiler A, Caborn DN, Brown CH Jr, Johnson DL (2000) Graft fixation in cruciate ligament reconstruction. Am J Sports Med 28(5):761-774

4. Cain EL, Phillips BB, Charlebois SZ, Azar FM (2005) Effect of tibial tunnel dilation on pullout strength of semitendinosusgracilis graft in anterior cruciate ligament reconstruction. Orthopedics 28(8):779-783

5. Drogset JO, Straume LG, Bjørkmo I, Myhr G (2011) A prospective randomized study of ACL-reconstructions using bonepatellar tendon-bone grafts fixed with bioabsorbable or metal interference screws. Knee Surg Sports Traumatol Arthrosc 19(5):753-759

6. Drogset JO, Grontvedt T, Myhr G (2006) Magnetic resonance imaging analysis of bioabsorbable interference screws used for fixation of bone-patellar tendon-bone autografts in endoscopic reconstruction of the anterior cruciate ligament. Am J Sports Med 34(7):1164-1169

7. Emond CE, Woelber EB, Kurd SK, Ciccotti MG, Cohen SB (2011) A comparison of the results of anterior cruciate ligament reconstruction using bioabsorbable versus metal interference screws: a meta-analysis. J Bone Joint Surg Am 93(6):572-580

8. Frosch KH, Sawallich T, Schütze G, Losch A, Walde TA, Balcarek P, Konietschke F, Stürmer KM (2009) Magnetic resonance imaging analysis of the bioabsorbable Milagro interference screw for graft fixation in anterior cruciate ligament reconstruction. Strateg Trauma Limb Reconstr 2:73-79

9. George MS, Dunn WR, Spindler KP (2006) Current concepts review: revision anterior cruciate ligament reconstruction. Am J Sports Med 34(12):2026-2037

10. Halewood C, Hirschmann MT, Newman S, Hleihil J, Chaimski G, Amis AA (2011) The fixation strength of a novel ACL softtissue graft fixation device compared with conventional interference screws: a biomechanical study in vitro. Knee Surg Sports Traumatol Arthrosc 19(4):559-567

11. Harilainen A, Sandelin J (2009) A prospective comparison of 3 hamstring ACL fixation devices-Rigidfix, BioScrew, and Intrafix-randomized into 4 groups with 2 years of follow-up. Am J Sports Med 37(4):699-706

12. Hoeher J, Moller HD, Fu FH (1998) Bone tunnel enlargement after anterior cruciate ligament reconstruction: fact or fiction? Knee Surg Sports Traumatol Arthrosc 6(4):231-240

13. Hunt $P$, Rehm $O$, Weiler A (2006) Soft tissue graft interference fit fixation: observations on graft insertion site healing and tunnel remodeling 2 years after ACL reconstruction in sheep. Knee Surg Sports Traumatol Arthrosc 14(12):1245-1251

14. Johnson D, Harner CD, Maday MG (1994) Revision anterior cruciate ligament surgery. In: Fu F, Harner CD, Vince KG (eds) Knee surgery. Williams \& Wilkins, Baltimore, pp 877-895

15. Kocher MS, Steadman JR, Briggs KK, Sterett WI, Hawkins RJ (2004) Relationships between objective assessment of ligament stability and subjective assessment of symptoms and function after anterior cruciate ligament reconstruction. Am J Sports Med 32(3):629-634

16. Logan M, Williams A, Myers P (2003) Is bone tunnel osseointegration in hamstring tendon autograft anterior cruciate ligament reconstruction important? Arthroscopy 19(8):E1-E3
17. Marks P, O'Donnell S, Yee G (2008) A pilot clinical evaluation comparing the Mitek bone-tendon-bone cross pin and bioabsorbable screw in anterior cruciate ligament reconstruction fixation, a randomized double blind controlled trial. Knee 15(3): $168-173$

18. Marshall JL, Fetto JF, Botero PM (1977) Knee ligament injuries. A standardized evaluation method. Clin Orthop Relat Res 123:115-129

19. Musahl V, Voos J, O’Loughlin PF, Stueber V, Kendoff D, Pearle AD (2010) Mechanized pivot shift test achieves greater accuracy than manual pivot shift test. Knee Surg Sports Traumatol Arthrosc 18(9): 1208-1213

20. Myers P, Logan M, Stokes A, Boyd K, Watts M (2008) Bioabsorbable versus titanium interference screws with hamstring autograft in anterior cruciate ligament reconstruction: a prospective randomized trial with 2-years follow-up. Arthroscopy 24(7):817-823

21. Noyes FR, Butler DL, Grood ES et al (1984) Biomechanical analysis of human grafts used in knee-ligament repairs and reconstructions. J Bone Joint Surg Am 66(3):344-352

22. Radford MJ, Noakes J, Read J, Wood DG (2005) The natural history of a bioabsorbable interference screw used for anterior cruciate ligament reconstruction with a 4-strand hamstring technique. Arthroscopy 21(6):707-710

23. Shen C, Jiang SD, Jiang LS, Dai LY (2010) Bioabsorbable versus metallic interference screw fixation in anterior cruciate ligament reconstruction: a meta-analysis of randomized controlled trials. Arthroscopy 26(5):705-713

24. Singhal MC, Holzhauer M, Powell D, Johnson DL (2008) MRI evaluation of the tibial tunnel/screw/tendon interface after ACL reconstruction using a bioabsorbable interference screw. Orthopedics 31(6):575-579

25. Tegnander A, Engebretsen L, Bergh K (1994) Activation of the complement system and adverse effects of biodegradable pins of polylactic acid (Biofix) in osteochondritis dissecans. Acta Orthop Scand 65(4):472-475

26. Tegner Y, Lysholm J (1985) Rating systems in the evaluation of knee ligament injuries. Clin Orthop Relat Res 198:43-49

27. Warden WH, Chooljian D, Jackson DW (2008) Ten-year magnetic resonance imaging follow-up of bioabsorbable poly-L-lactic acid interference screws after anterior cruciate ligament reconstruction. Arthroscopy 24(3):371-373

28. Weninger P, Zifko B, Liska M, Spitaler R, Pelinka H, Hertz H (2008) Anterior cruciate ligament reconstruction using autografts and double biodegradable femoral cross-pin fixation: functional, radiographic and MRI outcome after 2-year minimum follow-up. Knee Surg Sports Traumatol Arthrosc 16(11):988-995

29. Zantop T, Weimann A, Rümmler M, Hassenpflug J, Petersen W (2004) Initial fixation strength of two bioabsorbable pins for the fixation of hamstring grafts compared to interference screw fixation: single cycle and cyclic loading. Am J Sports Med 32(3): 641-649

30. Zantop T, Weimann A, Schmidtko R, Herbort M, Raschke MJ, Petersen W (2006) Graft laceration and pullout strength of softtissue anterior cruciate ligament reconstruction: in vitro study comparing titanium, poly-d, l-lactide, and poly-d, 1-lactide-tricalcium phosphate screws. Arthroscopy 22(11):1204-1210

31. Zantop T, Weimann A, Wolle K, Musahl V, Langer M, Petersen W (2007) Initial and 6 weeks postoperative structural properties of soft tissue anterior cruciate ligament reconstructions with crosspin or interference screw fixation: an in vivo study in sheep. Arthroscopy 23(1):14-20 